\title{
Anke Teigeler (Hrsg.): Ins Nichtwissen eintreten. Discipline of Authentic Movement
}

\author{
Reichert Verlag, 2018, Wiesbaden, 292 Seiten, 29,95€(D)
}

W as geschieht von dem Moment an, wenn wir uns einlassen und stehen, wenn wir lauschen und uns der unermesslichen Weite öffnen?

Dieser Frage widmen sich 18 Autorlnnen in der vorgelegten Sammlung in Aufsätzen, Bildern und Gedichten. Neben einem Interview und vier erstmals ins Deutsche übersetzten Beiträgen von Janet Adler, der Begründerin der Discipline of Authentic Movement/des Übungswegs Authentischer Bewegung, finden sich hier zwei Artikel der Herausgeberin Anke Teigeler und vielfältige Erfahrungsberichte von Lehrenden und langjährig Praktizierenden des Übungsweges.

Für die Herausgeberin war der Impuls, sich an die Veröffentlichung dieses Buches heranzuwagen, ein 2014 nur online veröffentlichter Artikel von Adler, in dem sie die von ihr unterrichtete Weise der authentischen Bewegung als Discipline of Authentic Movement benennt und als spirituellen Übungsweg vorstellt. Damit kennzeichnete sie diese im Körper verankerte, mystische Praxis „als eigenständige Strömung mit einem charakteristischen Schwerpunkt innerhalb des wachsenden Gesamtfeldes Authentic Movement“, wie Teigeler in der Einführung zum Buch schreibt (S.7).

Man kann mit Fug und Recht sagen, dass hier ein Grundlagenwerk entstanden ist, in dem besonders dieser spezielle „Ast am Baum“ der authentischen Bewegung, nämlich die Discipline of Authentic Movement, erstmals genauer beschrieben wird.

Als Wurzeln des Übungsweges dürfen gelten: Heilung, Tanz und Spiritualität/Mystik. In der Verbindung und gegenseitigen Befruchtung dieser drei Bereiche entfaltet sich das weite Potential der Discipline of Authentic Movement.
Dabei wird Mystik verstanden als unmittelbar im Körper - genau an der Schwelle zwischen Unbewusstem und Bewusstem - erlebbare, direkte Erfahrung.

An vielen Stellen im Buch wird folgende Aufgabe mit viel Kreativität, Kompetenz, tiefem Erfahrungswissen und der nötigen Sensibilität bewältigt: das eigentlich Unaussprechliche und mit Worten nur schwer Fassbare in Worte zu fassen und damit eine begehbare und miteinander teilbare Brücke zwischen Körper und Bewusstheit zu schaffen.

Das Buch ist in fünf Kapitel gegliedert: Zunächst wird sich dem Thema allgemein angenähert, wobei der Leser eine vielfältige und prägnante Veranschaulichung des Übungswegs erhält, die ihm zudem Einblicke in wesentliche, den Weg kennzeichnende Aspekte, Prozesse und Strukturen gewährt.

Hier erfahren wir zum Beispiel, dass Adler die Grundform der Discipline of Authentic Movement (die Arbeit zu zweit) sowohl bei Freud wie bei C.G. Jung verwurzelt sieht, dass aber ihr Weg, direkte Erfahrung zu begreifen, möglicherweise östlich orientiert ist und sie sich fragt, ob der Übungsweg die weibliche Form des Zen ist, denn entscheidend für diese Form der körperbasierten Achsamkeitspraxis ist, dass sie in Beziehung praktiziert wird.

In diesem Kapitel findet sich auch ihr Artikel „Die Mandorla und der Übungsweg Authentic Movement“, der dem Leser anhand des Bildes der Mandorla den Übungsweg erklärt und die Entwicklung des im Körper verankerten ZeugInnenbewusstseins in dessen Mittelpunkt rückt. Hier wird auch bereits deutlich gemacht, was 
den Übungsweg als zeitgenössische Gewahrseinspraxis ausweist:

„Eine im Körper verankerte mystische Praxis, die die Möglichkeit einer Wandlung zu neuen Wegen des Wissens einlädt, muss das Licht und den Schatten der Persönlichkeit mit einschließen, um in das heutige Leben integriert zu werden.“ (S.26)

Einen ersten Geschmack von dem feinfühligen Umgang mit Sprache, der sowohl in der Discipline of Authentic Movement wie in dem vorliegenden Buch praktiziert wird, liefert der Artikel „Authentische Bewegung als Weg“, in dem der Leser dazu aufgefordert wird, nicht nur den Text, sondern auch die leeren Zeilen dazwischen zu lesen, weil dies „eine andere Erfahrung ermöglichen (kann), als einen Satz nach dem anderen zu lesen." (S.38)

Im zweiten Kapitel geht es um das heilsame Potential des Übungsweges. Dabei werden Einflüsse und Wirkungen der authentischen Bewegung auf psychotherapeutisches Arbeiten betrachtet, wie in dem Artikel „Vertrauen ist stärker als Angst“. Im Hinblick auf eine sich gegenseitig erweiternde Wechselwirkung wird hier westliches psychotherapeutisches Wissen durch die innere Haltung von Mitgefühl und Präsenz vertieft und mit der verkörperten direkten Erfahrung des Numinosen verbunden. Spirituelles Erlebens wird aber auch als Ressource im Kontext traumatischer Erfahrungen unter Einbezug aktueller Forschungen und Methoden untersucht.

Zuletzt findet sich in diesem Kapitel eine Beschreibung des als heilsam empfundenen Prozesses, wie er sich bei einer Praktizierenden auf dem Weg unter der Diagnose Brustkrebs im Laufe von zwei Jahren entfaltet hat. Hier wird auf dem Übungsweg erfahren, wie „die Weisheit unseres Leibes durch Bewegung in die Welt gesetzt" wird. (S.131)

Im folgenden dritten Kapitel werden die Landschaften des schöpferischen Potentials des Übungsweges gleichsam durchwandert. Der Leser kann visuelle Eindrücke von „Verkör- perlichungen" der auf dem Übungsweg durchlebten Prozesse anschauen, die als bildnerisches Material ins Buch, ins Sichtbare, gegeben wurden. Er lernt außerdem etwas über die verschiedenen Gestaltungsformen des Kreises als Ritual in der authentischen Bewegung, wie er die Konzentration auf das Wesentliche fördert: als "Segen einer liebevollen Hinwendung in diese Welt, zu mir und den anderen“.

Sprache taucht an dieser Stelle in Form von „Körperworten“ auf, als Poesie, die aus der direkten Erfahrung geboren wird. Hier eröffnet sich dem Leser ein besonders unmittelbarer Blick auf die Erlebenswelt des Übungswegs.

Im vierten Kapitel erschließt sich uns das spirituelle Potential des beschriebenen Weges. Hier werden vergleichende Betrachtungen angestellt zwischen der Discipline of Authentic Movement und anderen spirituellen Wegen, z.B. mit einem kontemplativen christlichen Erfahrungsweg oder mit Tai Ji Do als einem weiteren Weg, „das Unbenennbare zu erfahren und zu erkennen." (S. 202) Im Vergleich werden Zen und die Discipline of Authentic Movement einander gegenübergestellt. Dabei werden Praxis und Weitergabe beider Wege differenziert.

Das 5. Kapitel führt uns zu theoretischen Reflexionen. Hier wird die Reise der inneren Zeugin erforscht, ,das dynamische Mysterium individuellen Wachstums, möglich gemacht durch einfache respektvolle Beziehung." (S. 265). Dabei lassen sich Parallelen zwischen der Praxis der phänomenologischen Forschung und der Praxis des ZeugInnenbewusstseins in der Discipline of Authentic Movement erkennen. Außerdem finden sich zwei weitere Texte von Adler in diesem Kapitel: Texte, aus denen die ganze Reife des Körper-Wissens der Autorin spürbar wird und in denen sie ihre tiefen - auf dem Übungsweg gemachten - Erfahrungen mit dem geneigten Leser teilt. In diesem Kapitel wird auch die transformative Kraft dieser Arbeit vermittelt.

Das Buch ist ein mehr als gelungener Versuch, dem nachzugehen, was Erfahrungswis- 
sen sein kann, und zu zeigen, wie es gelingt, dieses zur Mehrung unseres kollektiven Wissenspools nicht nur überzeugend, sondern auch überprüfbar zusammenzutragen. Und letztlich ist diese Sammlung - einschließlich des Gedichtes auf der allerletzten Seite - auch ein kleines gelungenes Kunstwerk.

Für Menschen mit Interesse an spirituellen Fragen oder die - für sich oder ihre Klientlnnen - nach einer mystischen Praxis suchen, die in einem modernen Kontext praktiziert werden kann, also vor dem Hintergrund unter- schiedlichster spiritueller Orientierungen und im heutigen Wissen um psychologische, gruppenrelevante und therapeutische Aspekte des menschlichen Seins, bietet das Buch einen reichen Fundus an vielfältigen Erfahrungsberichten und tiefen Einsichten, um sich ein Bild von den vielschichtigen Möglichkeiten des Übungsweges Authentic Movement zu machen.

\section{Marion Reuter}

DOI 10.2378/ktb2018.art25d

\title{
Helga Krüger-Kirn / Bettina Schroeter (Hrsg.): Verkörperungen von Weiblichkeit. Gendersensible Betrachtungen körperpsychotherapeutischer Prozesse
}

\author{
Psychosozial-Verlag, 2017, Gießen, 324 Seiten, 34,90€ (D)
}

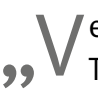
erkörperungen von Weiblichkeit“"ist ein Titel, der all diejenigen, die sich mit Körper und Psychotherapie befassen, neugierig macht. Sind die Lebenserfahrungen von Frauen anders im Körper verankert als die von Männern? Die 2013 in Marburg stattgefundene Tagung „Das andere Wissen“ der Deutschen Gesellschaft für Körperpsychotherapie (DGK) versuchte, dieser Frage nachzugehen, und befasste sich mit den spezifisch weiblichen Bedingungen von Körpererfahrung, Körperwahrnehmung und Körperausdruck. Die Herausgeberinnen des Tagungsbandes, Bettina Schroeter und Helga Krüger-Kirn, betonen in ihrer Einleitung die gesellschaftliche Relevanz der Thematik, die über die individuelle Perspektive in den soziokulturellen Raum hinein wirkt.

Der Band enthält alle Tagungsbeiträge. Das Inhaltsverzeichnis ist in drei Abschnitte unterteilt, zu denen jeweils mehrere Beiträge gehören. Auf diese Weise haben die LeserInnen dieser Publikation schon durch das Inhaltsverzeichnis einen Kompass, der durch die Ta- gungsthemen führt. Die Themen sind unter folgenden Großtiteln zusammengefasst: I Frauenkörper - Sprache - Körperwissen, II Schönheit Sexualität - Mutterschaft, III Phänomene moderner Weiblichkeit im körperpsychotherapeutischen Prozess.

Der erste Abschnitt enthält eher konzeptionelle Beiträge. Bettina Schroeter geht es darum, dass Frauen den Zugang zu ihrem Körper finden, um sich in ihrem „eigenen Körper zu beheimaten“ (S.36). Helga Krüger-Kirn zeigt in ihrem Beitrag, dass das „emanzipatorische Potential der Körperpsychotherapie (...) in der Sichtbar- und Sprechbarmachung weiblicher Körperlichkeit“ liegt (S.59).

Susanne Maurer warnt davor, Körperwahrnehmungen in einer undifferenzierten Weise zu deuten oder zu verstehen. Der Artikel von Sabine Schrem beschreibt ein Projekt, in dem Körperpsychotherapeutinnen sich auf die 